\title{
Depression and autoimmune thyroiditis - their relationship and the effects of treating psychiatric and thyroid disorders on changes in clinical and biochemical parameters including BDNF and other cytokines.
}

\author{
Zofia Kotkowska ${ }^{1}$, Dominik Strzelecki 2,*
}

\author{
Department of Affective and Psychotic Disorders, Central Clinical Hospital, ul. Czechosłowacka 8/10, 92-238 \\ Łódź, Medical University of Łódź, Poland \\ * Correspondence: dominik.strzelecki@umed.lodz.pl; Tel.: +48426757371
}

\begin{abstract}
Various autoimmune diseases, including autoimmune hypothyroidism (AHT), are associated with a higher risk of developing mood disorders throughout life. Depression is accompanied by the changes in the levels of inflammatory and trophic factors, including interleukines (IL-1beta, IL-2, IL-6), interferon alpha (IFN-alpha), tumor necrosis factor alpha (TNF-alpha), C-reactive protein (CRP) and brain derived neurotrophic factor (BDNF). Similar disturbances in the cytokine profile are seen in AHT patients and their relatives. Disclosure of the relationship between the coexistence of depression and autoimmune subclinical thyroiditis indicates that the pathomechanism of depression may be related to the changes in the immune system, it is possible that both conditions may be caused by the same immune processes. The above hypothesis is indirectly supported by the observations that the treatment with both antidepressants and levothyroxine leads to a decrease in the levels of proinflammatory cytokines with an increase in BDNF concentrations, simultaneously correlating with an improvement in the clinical parameters. However, so far there are no long-term studies determining the causal relationship between depression, thyroid autoantibodies, and cytokine profile, which could bring us closer to understanding the interrelationships between them and facilitate the use of an adequate pharmacotherapy, not necessarily psychiatric. We consider the above issues insufficiently investigated but of great importance. This article is an overview of the available literature as well as an introduction to our research project.
\end{abstract}

Keywords: depression, chronic autoimmune thyroiditis; BDNF

\section{Introduction}

The relationship between thyroid function and depression has been known for a long time [1-3]. It was first described in 1825 by Parry, who noted an increased number of "nerve strokes" in thyroid disease. Seagull in 1873 showed a link between myxoedema and psychosis, which was confirmed in 1888 by the Committee of the Clinical Society. In 1949 Asher introduced the term "myxedema madness" to describe mental state changes in patients with hypothyroidism [4]. It is currently well known that thyroid dysfunction can significantly affect the mental state, including emotions and cognitive functions. Both the excess and the deficiency of thyroid hormones can cause mood disorders, including depressive disorders, which can be usually resolved with an appropriate treatment of dysthyreosis. Depression may, in turn, be accompanied by various degrees of thyroid dysfunction. An overt hypothyroidism is present in 1-4\% of patients with affective disorders, while subclinical hypothyroidism occurs in $4-40 \%$ of this population. According to Boswell, the frequency of depressive symptoms in patients with hypothyroidism reaches $50 \%$ [5], while depression of significant clinical severity occurs in over $40 \%$ of people suffering from hypothyroidism [6]. What is practically very important, and what prompted us to 
undertake the research, the depressive states associated with broadly understood thyroid dysfunction are usually at least partially resistant to antidepressive treatment, with the causal treatment being the key here. Thyroid hormones are also usually recommended as an adjunctive therapy in the treatment of depression in treatment algorithms [7].

\section{Autoimmune hypothyroidism (AHT) and depression}

AHT is a progressive disease of the thyroid gland. Dense lymphocytic infiltration covering the gland is involved in the pathogenesis of this type of chronic thyroiditis [8]. Activated B cells produce antibodies against several major thyroid antigens. AHT is characterized by a combination of typical clinical features (please see table) [9], increased serum TSH levels, decreased fT4 levels, presence of antibodies to thyroid antigens, and decreased ultrasonographic echogenicity of the thyroid parenchyma [10].

Table. Autoimmune hypothyroidism - symptoms, assessment and diagnostic findings

\begin{tabular}{ccc}
\hline Symptoms & Assessment Findings & Diagnostic Findings \\
\hline Depression & Dry, coarse skin & Hyponatremia \\
\hline Fatigue & Reduced body and scalp hair & Macrocytic anemia \\
\hline Weight gain & Dull facial expression & Decreased memory \\
\hline Constipation & Bradycardia & Hyperprolactinemia \\
\hline Muscle cramps, arthralgias & Goiter & Elevated creatine kinase level \\
\hline Menorrhagia & Macroglossia & Pituitary gland enlargement \\
\hline Infertility & Ascites & Delayed bone age \\
\hline Sexual dysfunction & Galactorrhea & Hypercholesterolemia \\
\hline Cold intolerance & Slow relaxation of tendon & \\
\hline Carpal tunnel syndrome & reflexes & \\
\hline Sleep disorders & Nonpitting edema of lower & \\
\hline
\end{tabular}

AHT is the most common organ autoimmune disorder, with an estimated prevalence at approximately $2 \%$, women suffer more frequently. Thyroid peroxidase (TPO) is the main autoantigen, and antibodies to TPO (Anti-TPO) are present in almost all AHT patients and may precede onset of the clinical phase by up to several years. Subclinical AHT (with the presence of Anti-TPO, elevated TSH and normal fT4 levels) is more frequent and affects approximately $9 \%$ of general population [10].

Depression, including severe depression, is more common in patients with euthyroid chronic AHT comparing to subjects without this condition [8]. Frequent simultaneous presence of depressive and anxiety disorders in patients with Hashimoto's disease (over $90 \%$ of AHT) in the euthyroid stage has also been confirmed [11]. A large Danish epidemiological study has shown that various autoimmune diseases, including AHT, are associated with a higher occurence of mood disorders throughout life [12]. It has been shown that it is not only the decreased level of thyroid hormones that determines the occurrence of mood disorders, we also know that even in patients with normal thyroid function and elevated Anti-TPO antibodies, their presence correlates with a higher risk of anxiety and mood disorders [13]. An increased levels of anti-thyroid antibodies has been documented in $20 \%$ of patients with depression, while the incidence in the general population ranges from 5 to $10 \%[14,15]$. In patients with bipolar disorder, regardless of the use of lithium (having "anti-thyroid" properties), the presence of Anti-TPO and AHT is also more frequent $[16,17]$. Also, the offspring of people with bipolar disorder have a higher incidence of Anti-TPO, even if they do not have mental disorders [18,19]. There are also data showing an increased level of Anti-TPO and antibodies blocking the TSH receptor in patients 
with depressive disorders without thyroid dysfunction, but who were resistant to antidepressant treatment [20]. Several hypotheses are proposed to explain the above observations. Decrease in the secretion of thyroid hormones is interpreted as a result of dysregulation of TSH secretion circadian rhythm (physiologically TSH levels are higher at night). This suggests that some depressed patients may have central hypothyroidism (pituitary and hypothalamus dependent - secondary and tertiary, respectively). Moreover, sleep deprivation used in the treatment of depression leads to the restoration of the nocturnal rise in TSH levels and, consequently, an increase in fT3 and fT4 levels. According to another hypothesis, autoimmune thyroid diseases are associated with the hypothalamic-pituitary-adrenal axis and are mediated by changes in the concentration of pro-inflammatory and anti-inflammatory cytokines. TNF-alpha, IL-1 and IL-6 (all pro-inflammatory agents) increase the release of CRH (corticoliberin) and AVP (arginine vasopressin) leading to increase of glucocorticosteroids (GCS) secretion from the adrenal cortex. Chronic high GCS levels induce receptor resistance to GCS, subsequently causing further increase in their secretion and also disturbances in the functioning of the hypothalamic-pituitaryadrenal axis increasing the susceptibility to autoimmune processes and depression [21,22]. Next hypothesis connects the frequent postpartum recurrence of depression with the end of immune tolerance present in pregnancy [23,24]. Unfortunately, currently there are no studies showing a relationship between postpartum thyroiditis and postpartum depression and convincingly explaining the etiopathogenetic relationships but indicate the existence of common elements of the immune pathogenesis of both AHT and mood disorders.

The results of the summary [25] on depression, anxiety and AHT indicate, that in the United States the prevalence of AHT is 4-13\%, AHT more often affects women, its occurrence increases with age reaching $20 \%$ in the group of older women. Prevalence of depression reached $6.6 \%$, and anxiety disorders $18.1 \%$ in this study. It was also found that depression occurs in $16.8 \%$ of patients suffering from AHT, while the criteria for anxiety disorders were met by $35.7 \%$ of patients.

The study of the relationship between morphological changes in the central nervous system in AHT patients with hypothyroidism using positron emission tomography (PET) did not show any direct relationship between specific changes caused by AHT and the development of depressive disorders [2,26].

\section{Inflammatory processes, growth factors and cytokines}

Common etiological basis for both thyroid autoimmunity and mood disorders are also seen in similar changes of growth and differentiation in the hematopoietic and neuronal system cells and similar changes in the cytokine profile [10]. Numerous studies indicate that depression activates the inflammatory response system through increased production of pro-inflammatory cytokines such as IL-1beta, IL-2, IL-6, IFN-alpha, TNF-alpha and their receptors (IL-6R, IL-1RA) [27]. Stress induces production of pro-inflammatory cytokines leading to neuroendocrine and neurotransmitter changes resembling symptoms of depression. IFN-alpha immunotherapy (e.g., used in hepatitis C) often causes depressive symptoms and autoimmune thyroid disorders with the appearance of anti-thyroid peroxidase (Anti-TPO) and anti-thyroglobulin (anti-TG) antibodies [28].

The presence of acute phase proteins and cytokines may be associated with inflammation within the brain. Peripherally produced cytokines can cross the blood-brain barrier $[29,30]$, and after crossing it, they are able to participate in stress response modulation and regulation of neurogenesis [28]. One of their potential mechanisms of action on mood is the attenuation of neurogenesis within hippocampal neurons, which is believed to be a key mechanism in the pathophysiology of depression and its treatment [31-33]. The weakening of neurogenesis may, over time, contribute to the reduction of the gray matter volume in hippocampus, often observed in depression [34]. It is still unclear whether the presence of acute phase proteins may be the cause, consequence or only accompanying 
the depression.

A study conducted in patients with hypothyroidism after thyroidectomy due to cancer showed increased levels of the pro-inflammatory cytokines IL-6, IL-10. IL-17, TNF-alpha and CRP. Levothyroxine therapy (used in hypothyroidism) resulted in a decrease in the level of these cytokines, but their levels were still higher than in healthy subjects [35]. In patients treated with levothyroxine, a simultaneous reduction in the level of pro-inflammatory cytokines and an increase in the levels of anti-inflammatory cytokines has been also demonstrated [36]. Interestingly, primary AHT is characterized by increased values of pro-inflammatory cytokines such as IL-2, IL-6, IL-15, TNF-alpha and CRP [7,36-38]. During treatment with levothyroxine, a significant decrease in the levels of IL-1, IL-2, IL6, IL-12, IFN-gamma, TNF-alpha and a significant increase in IL-10 (anti-inflammatory cytokine) was observed $[7,36]$. In the Polish study the incidence of mild and moderate depression among patients with hypothyroidism was initially 57\%. After 6 months of levothyroxine therapy and achieving euthyroidism nearly half of the group (42\%) remitted depressive symptoms [7].

Use of selective serotonin reuptake inhibitors (SSRI), our most frequently used group of antidepressants, reduces promoting-depression effect of pro-inflammatory cytokines. Fluoxetine has been shown to reduce the expression of IL-1beta, IL-6, and TNF-alpha, but interestingly subsequently increasing the level of IL-10 [39]. Desipramine (tricyclic antidepressant, TCA) reduces levels of TNF-alpha in the hippocampus and brainstem [40], and its clinical efficacy has been associated with its ability to alter the sensitivity of noradrenergic neurons to TNF-alpha [41]. In conclusion, studies evaluating the effects of SSRIs and other antidepressive drugs indicate that inflammatory factors contribute to the pathogenesis of depression, and that various antidepressants have ability to reduce the release of pro-inflammatory cytokines. It is worth noting, that preliminary data suggest that antiinflammatory drugs may be useful in mood disorders - it has been reported that in patients treated with rofecoxib and celecoxib, the depressive symptoms improvement was more pronounced than in the group not treated with these drugs [42,43]. This effect at least partially may be related to the analgesic effect of the COX-2 inhibitors, but studies on rats showed that use of rofecoxib leads to serotonin increase in frontal, parietal and temporal cortex, which may indicate their other-than-analgesic mechanism of action [42]. Even if not all depressive states have an inflammatory etiology, according to the available data there is possibly a separate subtype of depression of inflammatory origin or manifestation of inflammatory process is able to clinically mimics depression. Biological anti-inflammatory drugs, including anti-TNF-alpha antibodies as e.g., infliximab, adalimumab and golimumab potentially may be useful to treat those types of depression. Those particles have an ability to reduce severity of inflammation (e.g., in rheumatic diseases, psoriasis and Crohn's disease) very effectively. However, the conclusions from limited depression trials are ambiguous, although patients with high initial levels of pro-inflammatory cytokines may have more benefit with this treatment. [44,45]. Tocilizumab studies show completely different results, this IL-6 receptor blocker exacerbates depressive symptoms in haematological patients [46]. Interestingly, hallucinogens (increasingly studied in depression) and bupropion have the ability to block TNF-alpha or decrease its production [47].

Scarce data from studies concerning the thyroid gland function during use of TNF-alpha antibodies showed also positive results [48]. We need to underline here that depression (also with increased suicidal risk) and thyroiditis (new onset or its exacerbation) are among common side effects of TNF-alpha blockers.

Studies involving relatives of patients with AHT force even deeper reflections about common origin of both ATH and depression. Euthyroid women being relatives of AHT patients have abnormal serum levels of hematopoietic and neural growth and differentiation factors important in etiology of depression - BDNF (Brain-derived neurotrophic factor), IGFBP-2 (insulin-like growth factor binding protein), EGF (epidermal growth factor) and SCF (stem cell factor) [10,49-51]. 


\section{Brain-derived neurotrophic factor (BDNF) and depression, AHT and gender}

BDNF needs a broader description as a crucial element in current understanding of development and dynamics of depressive symptomatology. This particle belongs to the group of neurotrophins, proteins synthesized in the cells of the central and peripheral nervous system and involved in the development, function and protection of nerve cells. It regulates many processes in our body, including the development and growth of neurons, inhibiting apoptosis, promoting neurogenesis, neuroregeneration, and stimulating the formation of dendritic connections. BDNF participates in the regulation of neuronal plasticity related to learning and memory processes, influencing the process of synaptic long-term potentiation and long-term depression in the hippocampus. It also influences the development of serotonergic, dopaminergic, noradrenergic and cholinergic neurons. Dopaminergic neurons of the substantia nigra and striatum have been found to be the main source of BDNF secretion. BNDF easily cross the blood-brain barrier [52].

Preclinical studies show that stress reduces BDNF expression in the rat hippocampus, while a single two-sided direct infusion of BDNF into the rat hippocampus has an antidepressant effect in animal models of depression [53]. In humans, BDNF plays a significant role in the pathophysiology of mental disorders, especially depression, where its key role is undisputable. Patients with severe depressive symptoms show lower levels of BDNF compared to controls. Moreover, BDNF levels are correlated with the reduction of the hippocampal volume [54]. We also know that BDNF expression is lower in the prefrontal cortex and hippocampus of people who died committing suicide compared to the control group matched in terms of sex and age [55]. The use of sertraline, escitalopram and venlafaxine in the study by Matrisciano et al. resulted in significant clinical improvement despite varying effects on peripheral BDNF levels. A relationship was also found between an increase in BDNF serum levels and an improvement in the Hamilton Depression Rating Scale, thus indicating that a higher BDNF level in the blood serum corresponds to recovery [54]. It has been shown that BDNF levels were directly related to antidepressant responses, and people who responded well to treatment ( $>50 \%$ improvement in the scores of depression severity scales) had higher BDNF levels before treatment than non-responders, indicating that BDNF can be a potential predictor of the antidepressant response [56]. It was also confirmed that the concentration of BDNF and its changes are not rigidly correlated with improvement in depression, but that the level of BDNF generally increases during antidepressant treatment. Studies on rapid-acting antidepressant - ketamine - having different mechanism of action than SSRIs and TCAs, showed that the rapid antidepressant response after its administration is mediated by an increase in BDNF levels [57]. Research directly linking BDNF to thyroid disorders is scarce at present. Rats with earlyonset hypothyroidism exhibited lower levels of BDNF in the brain [58]. Preclinical studies conducted by Hung indicate that thyroxine protects against white matter damage by increasing the level of BDNF [59]. In clinical trials, higher TSH levels were associated with a more discrete increase in serum BDNF levels in depressed patients during antidepressant therapy [60], and lower baseline TSH levels correlated with greater improvements after fluoxetine and sertraline [61]. It has also been shown that observed clinical results were better among patients who had lower TSH levels during sertraline and triiodothyronine therapy [62]. On the other hand, the only study evaluating the effect of thyroid hormones on changes in BDNF in serum, plasma and platelets over the 3-month period of treatment with antidepressants in patients without thyroid disease showed that higher TSH levels correlated with a lower increase in serum levels of BDNF during antidepressive treatment. It was additionally indicated that with TSH increase BDNF concentrations decreased throughout the observation period [60]. In patients participating in this study, no such relationship was found between the levels of triiodothyronine (T3), thyroxine (T4), corticotropic hormone (ACTH), cortisol, prolactin (PRL), luteinizing hormone (LH), follicle stimulating hormone (FSH), estrogen and progesterone. A study evaluating similar relationships in patients with hypothyroidism and subclinical hypothyroidism has not 
been conducted so far.

Summarizing, it can be hypothesized that thyroid hormones may affect the response to antidepressant therapy through its influence on BDNF, but so far there are no sufficient data to confirm this hypothesis unequivocally.

Due to the epidemiology of depression and AHT, it is worth emphasizing that the relationship between the level of BDNF and depression may depend on gender. It has been shown that reduced BDNF values are more pronounced in women with depression, and long-term antidepressant use selectively raises its concentration in women. Therefore, it is possible that the more frequent occurrence of depression in women may be related to this mechanism and interactions with female sex hormones, especially since it has been shown that BDNF expression can be reduced by exogenous administration of estradiol [56].

\section{Summary}

People with higher risk of developing mood disorders, including depressive disorders, are at the same time more likely to develop autoimmune thyroid disease and vice versa, what may indicate a common pathogenetic roots. An abnormal profile of haemopoietic and neuronal growth factors, including BDNF, is observed in patients with mood disorders as well as in those at risk of developing ATH. Similar observations apply to the cytokine profile in patients with both diseases, in whom we observe an increase in the concentrations of pro-inflammatory interleukins, e.g., IL-1beta, IL-2, IL-6, and TNF-alpha. From the clinical perspective, the conclusion that seems to be of particular importance is that in euthyroid patients with autoimmune thyroiditis and elevated levels of anti-thyroid antibodies, the incidence and severity of depression is significantly higher [8]. Treatment with both antidepressants and levothyroxine leads to a decrease in the level of pro-inflammatory cytokines, an increase in the level of BDNF, correlating with an improvement in clinical parameters of depression. Hence one of the hypotheses that depression may belong to the spectrum of inflammatory and degenerative disorders [63].

For the above reasons, patients with depression and anxiety disorders should be tested for autoimmune hypothyroidism, and patients with AHT should be screened for psychiatric symptoms [25]. However, it is necessary to conduct long-term studies (as our team is planning now) to determine the causal relationship between depression, thyroid autoantibodies, and cytokine levels, which would help us bring us closer to understanding the interrelationships between them and facilitate the use of adequate pharmacotherapy, not necessarily psychiatric. At the same time, it would be possible to identify groups of higher risk for the occurrence of both frequent and very burdensome diseases, and from psychiatric perspective to help prevent the development of full-blown depression and finally to predict the therapeutic response to particular groups of drugs.

Author Contributions: Conceptualization, D.S.; methodology, D.S. and Z.K.; validation, D.S.; formal analysis, D.S. and Z.K; investigation, Z.K and D.S.; data curation, Z.K and D.S; writing - original draft preparation, Z.K and D.S; writing - review and editing, Z.K and D.S; visualization, Z.K and D.S.; supervision, D.S.; project administration, Z.K and D.S.; funding acquisition, D.S. All authors have read and agreed to the published version of the manuscript.

Funding: This research received no external funding.

Institutional Review Board Statement: Not applicable.

Informed Consent Statement: Not applicable.

Conflicts of Interest: The authors declare no conflict of interest. 


\section{References}

1. van Boxtel, M.P.J.; Menheere, P.P.C.A.; Bekers, O.; Hogervorst, E.; Jolles, J. Thyroid Function, Depressed Mood, and Cognitive Performance in Older Individuals: the Maastricht Aging Study. Psychoneuroendocrinology 2004, 29 (7), 891898.

2. Hage, M.P.; Azar, S.T. The Link between Thyroid Function and Depression. J. Thyroid. Res. 2012, 2012, 1-8.

3. Kirkegaard, C.; Faber, J. The Role of Thyroid Hormones in Depression. Eur. J. Endocrinol. 1998, 138 (1), 1-9.

4. Bhagwat, N.M.; Tayde, P.S.; Sharma, P.; Sharma, B.; Dalwadi, P.P.; Sonawane, A.; Subramanyam, A.; Chadha, M.; Varthakavi, P. K. Hypothyroidism and Depression: Are Cytokines the Link? Indian J. Endocrinol. Metab. 2017, 21 (6), 886.

5. Boswell, E.B.; Anfinson, T.H.; Nemeroff, C.B. Depression associated with endocrine disorder. In: Depression and Physical Illness (Perspectives in Psychiatry Volume 6) Edited by M. M. Robertson \& C. L. E. Katona. Chichester. John Wiley and Sons. 1996, 256-292.

6. Cleare, A.J.; McGregor, A.; O'Keane, V. Neuroendocrine Evidence for an Association between Hypothyroidism, Reduced Central 5-HT Activity and Depression. Clin. Endocrinol. 1995, 43 (6), 713-719.

7. Łojko, D.; Rybakowski, J. L-Thyroxine Augmentation of Serotonergic Antidepressants In Female Patients With Refractory Depression. J. Affect. Disord. 2007, 103, 253-256.

8. Kirim, S.; Keskek, S. Ö.; Köksal, F.; Haydardedeoglu, F. E.; Bozkirli, E.; Toledano, Y. Depression in Patients with Euthyroid Chronic Autoimmune Thyroiditis. Endocr. J. 2012, 59 (8), 705-708.

9. Dunn, D.; Turner, C. Hypothyroidism in Women. Nurs. Womens Health 2016, 20 (1), 93-8.

10. Massolt, E.T.; Effraimidis, G.; Korevaar, T.I.; Wiersinga, W.M.; Visser, W.E.; Peeters, R.P.; Drexhage, H.A. Aberrant Levels of Hematopoietic/Neuronal Growth and Differentiation Factors in Euthyroid Women at Risk for Autoimmune Thyroid Disease. PLOS ONE 2016, 11 (4).

11. Carta, M.G.; Hardoy, M.C.; Carpiniello, B.; Murru, A.; Marci, A.R.; Carbone, F.; Deiana, L.; Cadeddu, M.; Mariotti, S. A Case Control Study on Psychiatric Disorders in Hashimoto Disease and Euthyroid Goitre: Not Only Depressive but Also Anxiety Disorders are Associated with Thyroid Autoimmunity. Clin. Pract. Epidemiol. Ment. Health 2005, 1, 23.

12. Benros, M.E.; Waltoft, B.L.; Nordentoft, M.; Østergaard, S.D.; Eaton, W.W.; Krogh, J.; Mortensen, P.B. Autoimmune Diseases and Severe Infections as Risk Factors for Mood Disorders. JAMA Psychiatry 2013, 70 (8), 812.

13. Carta, M.G.; Loviselli, A.; Hardoy, M.C.; Massa, S.; Cadeddu, M.; Sardu, C.; Carpiniello, B.; Dell'Osso, L.; Mariotti, S. The Link between Thyroid Autoimmunity (Antithyroid Peroxidase Autoantibodies) with Anxiety and Mood Disorders in the Community: a Field of Interest for Public Health in the Future. BMC Psychiatry 2004, 4 (1).

14. Nemeroff, C.B.; Simon, J.S.; Haggerty, J.J.; Evans, D.L. Antithyroid Antibodies in Depressed Patients. Am. J. Psychiatry 1985, 142 (7), 840-843.

15. Joffe, R. T. Antithyroid Antibodies in Major Depression. Acta Psychiatr. Scand. 1987, 76 (5), 598-599.

16. Kupka, R.W.; Nolen, W.A.; Post, R.M.; McElroy, S.L.; Altshuler, L.L.; Denicoff, K. D.; Frye, M.A.; Keck, P.E.; Leverich, G.S.; Rush, A.J.; Suppes, T.; Pollio, C.; Drexhage, H.A. High Rate of Autoimmune Thyroiditis in Bipolar Disorder: Lack of Association with Lithium Exposure. Biol. Psychiatry 2002, 51 (4), 305-311.

17. Padmos, R.C.; Bekris, L.; Knijff, E.M.; Tiemeier, H.; Kupka, R.W.; Cohen, D.; Nolen, W.A.; Lernmark, Å.; Drexhage, H. A. A High Prevalence of Organ-Specific Autoimmunity in Patients with Bipolar Disorder. Biol. Psychiatry 2004, $56(7), 476-482$.

18. Vonk, R.; van der Schot, A.C.; Kahn, R.S.; Nolen, W.A.; Drexhage, H.A. Is Autoimmune Thyroiditis Part of the Genetic Vulnerability (or an Endophenotype) for Bipolar Disorder? Biol. Psychiatry 2007, 62 (2), $135-140$. 
19. Hillegers, M.H.J.; Reichart, C.G.; Wals, M.; Verhulst, F.C.; Ormel, J.; Nolen, W.A.; Drexhage, H.A. Signs of a Higher Prevalence of Autoimmune Thyroiditis in Female Offspring of Bipolar Parents. Eur. Neuropsychopharmacol. 2007, 17 (6-7), 394-399.

20. Fountoulakis, K.N.; Iacovides, A.; Grammaticos, P.; St Kaprinis, G.; Bech, P. Thyroid Function in Clinical Subtypes of Major Depression: an Exploratory Study. BMC Psychiatry 2004, 4 (1).

21. Tsigos, C.; Chrousos, G.P. Hypothalamic-Pituitary-Adrenal Axis, Neuroendocrine Factors and Stress. J. Psychosom. Res. 2002, 53 (4), 865-871.

22. Maes, M.; Meltzer, H.; Cosyns, P.; Suy, E.; Schotte, C. An Evaluation of Basal Hypothalamic-Pituitary-Thyroid Axis Function in Depression: Results of a Large-Scaled and Controlled Study. Psychoneuroendocrinology 1993, 18 (8), 607620.

23. Kuijpens, J.L.; Vader, H.L.; Drexhage, H.A.; Wiersinga, W.M.; van Son, M.J.; Pop, V.J. Thyroid Peroxidase Antibodies during Gestation Are a Marker for Subsequent Depression Postpartum. Eur. J. Endocrinol. 2001, 579-584.

24. Elenkov, I.J.; Wilder, R.L.; Bakalov, V.K.; Link, A.A.; Dimitrov, M.A.; Fisher, S.; Crane, M.; Kanik, K.S.; Chrousos, G.P. IL-12, TNF- $\alpha$, and Hormonal Changes during Late Pregnancy and Early Postpartum: Implications for Autoimmune Disease Activity during These Times. J. Clin. Endocrinol. Metab. 2001, 86 (10), 4933-4938.

25. Siegmann, E.-M.; Müller, H.H.; Luecke, C.; Philipsen, A.; Kornhuber, J.; Grömer, T.W. Association of Depression and Anxiety Disorders with Autoimmune Thyroiditis. JAMA Psychiatry 2018, 75 (6), 577.

26. Shojaie, M.; Eshraghian, A. Primary Hypothyroidism Presenting with Torsades De Pointes Type Tachycardia: a Case Report. Cases J. 2008, 1 (1).

27. Dowlati, Y.; Herrmann, N.; Swardfager, W.; Liu, H.; Sham, L.; Reim, E.K.; Lanctôt, K.L. A Meta-Analysis of Cytokines in Major Depression. Biol. Psychiatry 2010, 67 (5), 446-457.

28. Ajjan, R.A.; Weetman, A.P. Cytokines in Thyroid Autoimmunity. Autoimmunity 2003, 36 (6-7), 351-359.

29. Banks, W.A.; Kastin, A.J.; Broadwell, R.D. Passage of Cytokines across the Blood-Brain Barrier. Neuroimmunomodulation 1995, 2 (4), 241-248.

30. Banks, W.A.; Farr, S.A.; Morley, J.E. Entry of Blood-Borne Cytokines into the Central Nervous System: Effects on Cognitive Processes. Neuroimmunomodulation 2002, 10 (6), 319-327.

31. Malberg, J.E.; Eisch, A.J.; Nestler, E.J.; Duman, R.S. Chronic Antidepressant Treatment Increases Neurogenesis in Adult Rat Hippocampus. J. Neurosci. 2000, 20 (24), 9104-9110.

32. Saarelainen, T.; Hendolin, P.; Lucas, G.; Koponen, E.; Sairanen, M.; MacDonald, E.; Agerman, K.; Haapasalo, A.; Nawa, H.; Aloyz, R.; Ernfors, P.; Castrén, E. Activation of the TrkB Neurotrophin Receptor Is Induced by Antidepressant Drugs and Is Required for Antidepressant-Induced Behavioral Effects. J. Neurosci. 2003,23 (1), $349-357$.

33. Ekdahl, C.T.; Claasen, J.-H.; Bonde, S.; Kokaia, Z.; Lindvall, O. Inflammation Is Detrimental for Neurogenesis in Adult Brain. Proc. Natl. Acad. Sci. U S A 2003, 100 (23), 13632-13637.

34. Campbell, S.; Marriott, M.; Nahmias, C.; MacQueen, G. M. Lower Hippocampal Volume in Patients Suffering from Depression: A Meta-Analysis. Am. J. Psychiatry 2004, 161 (4), 598-607.

35. Baldissarelli, J.; Mânica, A.; Pillat, M.M.; Bagatini, M.D.; Leal, D.B.; Abdalla, F.H.; Morsch, V.M.; Ulrich, H.; Bornemann, C.P.; Chitolina Schetinger, M. R. Increased Cytokines Production and Oxidative Stress Are Related with Purinergic Signaling and Cell Survival in Post-Thyroidectomy Hypothyroidism. Mol. Cell. Endocrinol. 2020, 499, 110594.

36. Marchiori, R.C.; Pereira, L.A.; Naujorks, A.A.; Rovaris, D.L.; Meinerz, D.F.; Duarte, M.M.; Rocha, J.B. Improvement of Blood Inflammatory Marker Levels in Patients with Hypothyroidism under Levothyroxine Treatment. BMC Endocr. Disord. 2015, 15 (1). 
37. Mikoś, H.; Mikoś, M.; Obara-Moszyńska, M.; Niedziela, M. The Role of the Immune System and Cytokines Involved in the Pathogenesis of Autoimmune Thyroid Disease (AITD). Endokrynol. Pol. 2014, 65 (2), 150-155.

38. Konca Degertekin, C.; Aktas Yilmaz, B.; Balos Toruner, F.; Kalkanci, A.; Turhan Iyidir, O.; Fidan, I.; Yesilyurt, E.; Cakır, N.; Kustimur, S.; Arslan, M. Circulating Th17 Cytokine Levels Are Altered in Hashimoto's Thyroiditis. Cytokine 2016, 80, 13-17.

39. Chiou, S.-H.; Chen, S.-J.; Peng, C.-H.; Chang, Y.-L.; Ku, H.-H.; Hsu, W.-M.; Ho, L. L.-T.; Lee, C.-H. Fluoxetine UpRegulates Expression of Cellular FLICE-Inhibitory Protein and Inhibits LPS-Induced Apoptosis in HippocampusDerived Neural Stem Cell. Biochem. Biophys. Res. Commun. 2006, 343 (2), 391-400.

40. Reynolds, J.L.; Ignatowski, T.A.; Sud, R.; Spengler, R.N. An Antidepressant Mechanism of Desipramine Is to Decrease Tumor Necrosis Factor- $\alpha$ Production Culminating in Increases in Noradrenergic Neurotransmission. Neuroscience 2005, 133 (2), 519-531.

41. Reynolds, J.L.; Ignatowski, T.A.; Sud, R.; Spengler, R.N. Brain-Derived Tumor Necrosis Factor- $\alpha$ and Its Involvement in Noradrenergic Neuron Functioning Involved in the Mechanism of Action of an Antidepressant. J. Pharmacol. Exp. Ther. 2004, 310 (3), 1216-1225.

42. Müller, N.; Riedel, M.; Schwarz, M.J. Psychotropic Effects of COX-2 Inhibitors - A Possible New Approach for the Treatment of Psychiatric Disorders. Pharmacopsychiatry 2004, 37 (6), 266-269.

43. Müller, N.; Schwarz, M.J.; Dehning, S.; Douhe, A.; Cerovecki, A.; Goldstein-Müller, B.; Spellmann, I.; Hetzel, G.; Maino, K.; Kleindienst, N.; Möller, H.-J.; Arolt, V.; Riedel, M. The Cyclooxygenase-2 Inhibitor Celecoxib Has Therapeutic Effects in Major Depression: Results of a Double-Blind, Randomized, Placebo Controlled, Add-on Pilot Study to Reboxetine. Mol. Psychiatry 2006, 11 (7), 680-684.

44. Lamers, F.; Bot, M.; Jansen, R.; Chan, M.K.; Cooper, J.D.; Bahn, S, Penninx, B.W.J.H. Serum Proteomic Profiles of Depressive Subtypes. Transl. Psychiatry 2016, 6, e851.

45. Galvez-Contreras, A.Y.; Campos-Ordonez, T.; Lopez-Virgen, V.; Gomez-Plascencia, J.; Ramos-Zuniga, R.; Gonzalez-Perez, O. Growth Factors as Clinical Biomarkers of Prognosis and Diagnosis in Psychiatric Disorders. Cytokine Growth Factor Rev. 2016, 32, 85-96.

46. Benedetti, F.; Poletti, S.; Hoogenboezem, T.A.; Locatelli, C.; Ambrée, O.; de Wit, H.; Wijkhuijs, A.J.; Mazza, E.; Bulgarelli, C.; Vai, B.; Colombo, C.; Smeraldi, E.; Arolt, V.; Drexhage, H.A. Stem Cell Factor (SCF) is a Putative Biomarker of Antidepressant Response. J. Neuroimmune Pharmacol. 2016, 11 (2), 248-58.

47. Małczyńska, P.; Piotrowicz, Z.; Drabarek, D.; Langfort, J.; Chalimoniuk, M. [The Role of the Brain-Derived Neurotrophic Factor (BDNF) in Neurodegenerative Processes and in the Neuroregeneration Mechanisms Induced by Increased Physical Activity]. Postepy Biochem. 2019, 65 (1), 2-8.

48. Shirayama, Y.; Chen, A.C.-H.; Nakagawa, S.; Russell, D.S.; Duman, R.S. Brain-Derived Neurotrophic Factor Produces Antidepressant Effects in Behavioral Models of Depression. J. Neurosci. 2002, 22 (8), 3251-3261.

49. Matrisciano, F.; Bonaccorso, S.; Ricciardi, A.; Scaccianoce, S.; Panaccione, I.; Wang, L.; Ruberto, A.; Tatarelli, R.; Nicoletti, F.; Girardi, P.; Shelton, R. C. Changes in BDNF Serum Levels in Patients with Major Depression Disorder (MDD) after 6 Months Treatment with Sertraline, Escitalopram, or Venlafaxine. J Psychiatr. Res. 2009, 43 (3), 247254.

50. Dwivedi, Y.; Rizavi, H.S.; Conley, R.R.; Roberts, R.C.; Tamminga, C.A.; Pandey, G.N. Altered Gene Expression of Brain-Derived Neurotrophic Factor and Receptor Tyrosine Kinase B in Postmortem Brain of Suicide Subjects. Arch. Gen Psychiatry 2003, 60 (8), 804.

51. Huang, T.-L.; Lee, C.-T.; Liu, Y.-L. Serum Brain-Derived Neurotrophic Factor Levels in Patients with Major Depression: Effects of Antidepressants. J Psychiatr. Res. 2008, 42 (7), 521-525. 
52. Kavalali, E.T.; Monteggia, L.M. Synaptic Mechanisms Underlying Rapid Antidepressant Action of Ketamine. Am. J. Psychiatry 2012, 169 (11), 1150-1156.

53. Lasley, S.M.; Gilbert, M.E. Developmental Thyroid Hormone Insufficiency Reduces Expression of Brain-Derived Neurotrophic Factor (BDNF) in Adults but Not in Neonates. Neurotoxicol. Teratol. 2011, 33 (4), 464-472.

54. Hung, P.-L.; Huang, C.-C.; Huang, H.-M.; Tu, D.-G.; Chang, Y.-C. Thyroxin Treatment Protects Against White Matter Injury in The Immature Brain via Brain-Derived Neurotrophic Factor. Stroke 2013, 44 (8), $2275-2283$.

55. Baek, J.H.; Kang, E.-S.; Fava, M.; Mischoulon, D.; Nierenberg, A.A.; Lee, D.; Heo, J.-Y.; Jeon, H.J. Thyroid Stimulating Hormone and Serum, Plasma, and Platelet Brain-Derived Neurotrophic Factor during a 3-Month Follow-up in Patients with Major Depressive Disorder. J. Affect. Disord. 2014, 169, 112-117.

56. Gitlin, M.; Altshuler, L.L.; Frye, M.A.; Suri, R.; Huynh, E.L.; Fairbanks, L.; Bauer, M.; Korenman, S. Peripheral Thyroid Hormones and Response to Selective Serotonin Reuptake Inhibitors. J. Psychiatry Neurosci. 2004, 29, 383386.

57. Cooper-Kazaz, R.; van der Deure, W.M.; Medici, M.; Visser, T.J.; Alkelai, A.; Glaser, B.; Peeters, R.P.; Lerer, B. Preliminary Evidence That a Functional Polymorphism in Type 1 Deiodinase Is Associated with Enhanced Potentiation of the Antidepressant Effect of Sertraline by Triiodothyronine. J. Affect. Disord. 2009, 116 (1-2), 113-116.

58. Maes, M.; Bosmans, E.; Meltzer, H.Y.; Scharpé, S.; Suy, E. Interleukin-1 Beta: a Putative Mediator of HPA Axis Hyperactivity in Major Depression? Am. J. Psychiatry 1993, 150 (8), 1189-1193. 